\title{
latrogenic Infective Endocarditis With Septic Emboli: An Unusual Complication of Intracardiac Manipulation
}

\author{
Ndausung Udongwo ${ }^{\mathrm{a}, \mathrm{d}}$, Nusha Fareen ${ }^{\mathrm{a}}$, Temidayo Abe ${ }^{\mathrm{b}}$, Mihir Odak ${ }^{\mathrm{a}}$, \\ SmArif Saleh ${ }^{\mathrm{a}}$, Laith Zamelc
}

\begin{abstract}
Radiofrequency catheter ablation (RFA) is an effective treatment for recurrent symptomatic atrial fibrillation $(\mathrm{AF})$ despite medical therapy. Complications include cardiac tamponade, pulmonary vein stenosis, and rarely atrial-esophageal fistula (AEF). We present a case of a 77-year-old male patient with a history of persistent AF status post posterior left atrial isolation 50 days prior, who presented to the emergency department with bilateral lower extremity weakness ongoing for an hour. In addition, he reported a non-productive cough, chest discomfort, and dysphagia. He was hypertensive, febrile, tachycardic, and with tachypnea on initial assessment. Initial labs revealed leukocytosis with elevated troponin level. Brain imaging confirmed subacute infarcts suggestive of embolic disease. Tissue plasminogen activator was contraindicated. He was admitted for management of altered mental status and sepsis of an unknown origin. Due to associated chest discomfort and dysphagia, computed tomography (CT) scan of the chest with contrast obtained revealed a small focus of air between the posterior wall of the left atrium and the esophagus. A few hours later, he deteriorated with worsened hypoxic respiratory failure, altered mental status, requiring intubation and mechanical ventilation. Cardiothoracic surgery was consulted, and he was taken to the operating room for open-heart surgery due to concern for AEF. Peri-operatively, a left atrial fistula measuring $0.5 \mathrm{~cm}$ was identified and closed. Also, a fibrinous material $(3 \times 4 \mathrm{~cm})$ was identified and removed in the left inferior pulmonary vein. On post-operative day 1 , he underwent an upper endoscopy, and a $1 \mathrm{~cm}$ esophageal defect was closed. Biopsy from fibrinous material was positive for polymicrobial organisms (lactobacillus, candida, and prevotella), while blood cultures grew Streptococcus anginosus. Despite treatment with aggressive intravenous antibiotics, the patient deteriorated with poor
\end{abstract}

Manuscript submitted December 8, 2021, accepted January 18, 2022

Published online February 16, 2022

aDepartment of Internal Medicine, Jersey Shore University Medical Center, Neptune City, NJ 07753, USA

bDepartment of Internal Medicine, Morehouse School of Medicine, Atlanta, GA 30310, USA

'Department of Cardiology, Jersey Shore University Medical Center, Neptune City, NJ 07753, USA

${ }^{\mathrm{d} C}$ Corresponding Author: Ndausung Udongwo, Department of Internal Medicine, Jersey Shore University Medical Center, Neptune City, NJ 077543, USA.

Email: ndausung.udongwo@hmhn.org

doi: https://doi.org/10.14740/jmc3879 neurological function and ventilator dependency. A multidisciplinary team reached a consensus agreement for palliative measures. To our knowledge, this case represents one of the few cases documenting life-threatening infective endocarditis with septic emboli as a complication of AEF from RFA. Therefore, clinicians should have a high index of suspicion due to associated grave prognosis.

Keywords: Thromboembolism; Atrial fibrillation; Arrhythmia; Embolic stroke; Pulmonary vein isolation; Fistula; Iatrogenic; Atrialesophageal fistula

\section{Introduction}

Radiofrequency catheter ablation (RFA) is an effective treatment for recurrent symptomatic atrial fibrillation (AF) despite medical therapy. Complications include cardiac tamponade, pulmonary vein stenosis, gastrointestinal bleeding, new-onset arrhythmia, cardiac arrest, infections, thromboembolism (stroke), and rarely atrial-esophageal fistula (AEF) [1-4]. Symptoms of AEF vary from chest discomfort, cough, reflux symptoms, flu-like symptoms, to severe sepsis [5]. Usually, AEF occurs within 2 - 41 days following the procedure, and delay in management can lead to death $[2,5]$. Our team presents a case of a 77-year-old male patient with a history of persistent $\mathrm{AF}$ who presented with chest discomfort, dysphagia, and stroke 50 days after pulmonary vein isolation. Imaging findings confirmed AEF.

\section{Case Report}

\section{Investigations}

A 77-year-old male patient with a history of persistent AF status post posterior left atrial isolation 50 days prior presented to the emergency department with bilateral lower extremity weakness ongoing for an hour. In addition, he reported a non-productive cough, chest discomfort, and dysphagia. His other past medical history included: prostate cancer status post prostatectomy (in remission), asthma, hypertension, hyperlipidemia, and obstructive sleep apnea. He never smoked tobacco in the past, and his family history was unremarkable. His daily 


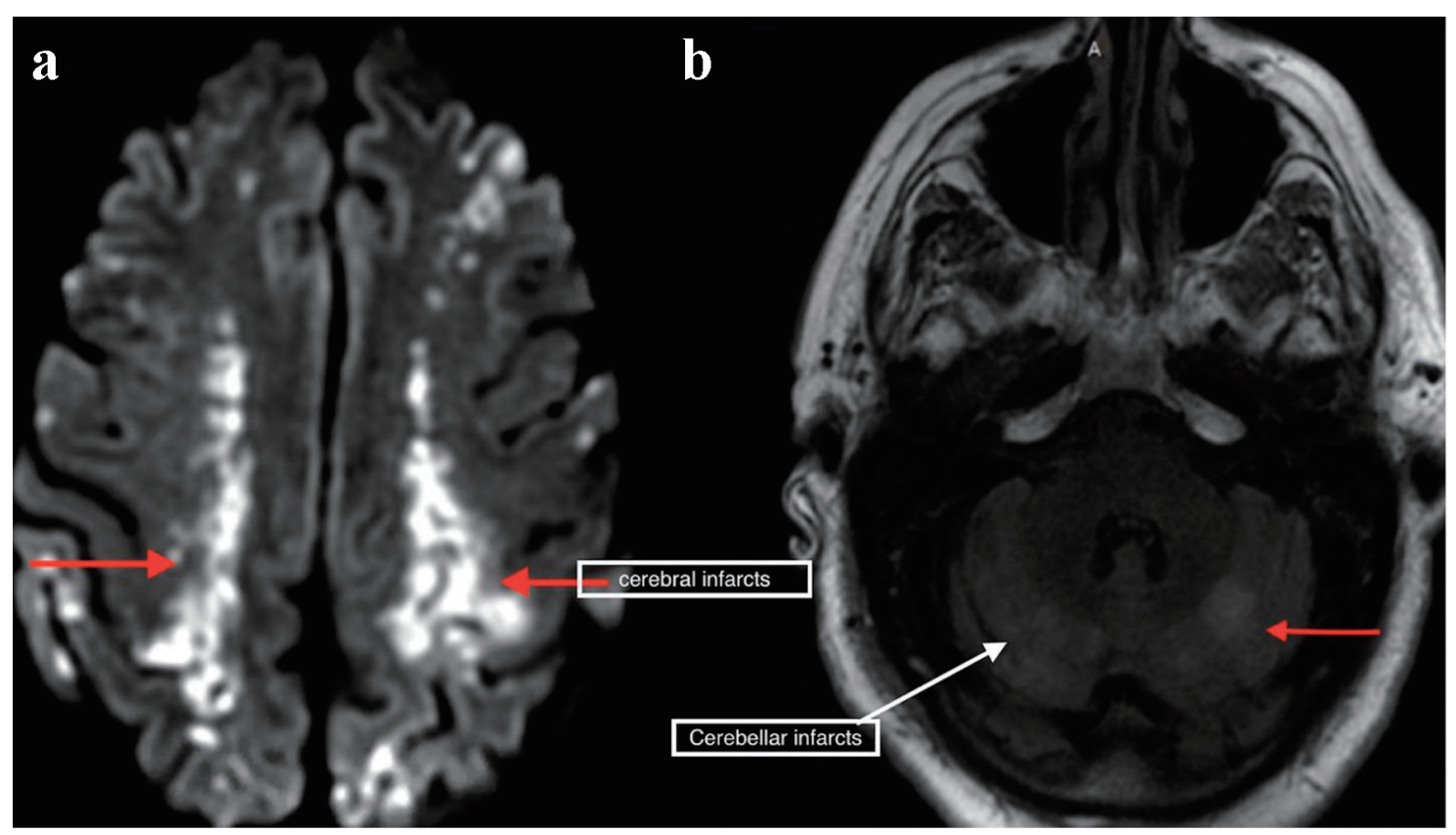

Figure 1. Magnetic resonance imaging of the brain showing bilateral cerebral (a) and cerebellar infarcts (b).

oral medications were metoprolol, diltiazem, aspirin, pravastatin, telmisartan-hydrochlorothiazide, apixaban, and albuterolipratropium. On initial assessment, vitals were blood pressure of 193/110 mm Hg; respiratory rate of 28 breaths per minute; heart rate of 135 beats per minute; temperature of $102.3^{\circ} \mathrm{F}$; oxygen saturation of $91 \%$ on room air. Glasgow Coma Scale (GCS) score was 14 (E4, V4, M6). On physical examination, he was alert and oriented only to person and place. There was a decreased light tough sensation and $4 / 5$ weakness in the bilateral lower extremities. Cranial nerves 2-12 were grossly intact. Cardiovascular examination was only significant for an irregular rhythm with tachycardia. Bilateral lungs sounds were audible on auscultation.

\section{Diagnosis}

Laboratory results revealed white blood cell count of $17.9 \times$ $10^{3} / \mu \mathrm{L}$ (normal value: $4.5-11.0 \times 10^{3} / \mu \mathrm{L}$ ), and troponin I of $1.76 \mathrm{ng} / \mathrm{mL}$ (normal value: $<0.04 \mathrm{ng} / \mathrm{mL}$ ). Other lab results showed glucose of $153 \mathrm{mg} / \mathrm{dL}$ (normal value: 70 - $99 \mathrm{mg} / \mathrm{dL}$ ), blood urea nitrogen of $29 \mathrm{mg} / \mathrm{dL}$ (normal value: 7 - $18 \mathrm{mg} / \mathrm{dL}$ ), creatinine of $1.34 \mathrm{mg} / \mathrm{dL}$ (normal value: $0.61-1.24 \mathrm{mg} / \mathrm{dL}$ ), lactate of $2.4 \mathrm{mmol} / \mathrm{L}$ (normal value: $0.2-2.0 \mathrm{mmol} / \mathrm{L}$ ), and creatinine kinase of 425 IU/L (normal value: 22 - 232 IU/L). An electrocardiogram showed irregularly irregular rhythm and no ST- or T-wave changes. Chest X-ray was unremarkable. Non-contrast CT scan of the head showed areas of low attenuation in the left parietal, temporal, and occipital region, with no acute intracranial hemorrhage. CT angiogram of the head and neck, CT perfusion neuro, and CT of the cervical spine without contrast were unremarkable. Magnetic resonance imaging of the brain with/without contrast revealed hyperintensity in both cerebral and cerebellar hemispheres, consistent with subacute infarcts suggestive of embolic disease (Fig. 1). Tissue plasminogen activator was contraindicated, as he was on apixaban. The patient was admitted for management of altered mental status and sepsis of an unknown origin. Due to associated chest discomfort and dysphagia, a CT scan of the chest with contrast obtained revealed a small focus of air between the posterior wall of the left atrium and the esophagus (Fig. 2). About $18 \mathrm{~h}$ later, he deteriorated with worsened hypoxic respiratory failure, altered mental status (GCS score of 6; E2, V2, M2), requiring intubation and mechanical ventilation.

\section{Treatment}

Cardiothoracic surgery was consulted, and he was taken to the operation room for open-heart surgery due to concern for AEF. Cardiopulmonary bypass was initiated, and the aorta was cross-clamped to avoid embolization. Cardioplegia was achieved. Peri-operatively, there was an area of induration along the atrioventricular groove, and the left atrial appendage was completely stuck to the ventricular wall. A left atrial fistula measuring $0.5 \mathrm{~cm}$ was identified and closed. Also, a fibrinous material $(3 \times 4 \mathrm{~cm})$ was identified and removed in the left inferior pulmonary vein. On post-operative day 1 , he underwent an upper endoscopy, and a $1 \mathrm{~cm}$ esophageal defect was closed. Biopsy from fibrinous material was positive for polymicrobial organisms (lactobacillus, candida, and prevotella), while blood cultures grew Streptococcus anginosus.

\section{Follow-up and outcomes}

Despite treatment with aggressive intravenous antibiotics, the patient deteriorated with poor neurological function and venti- 


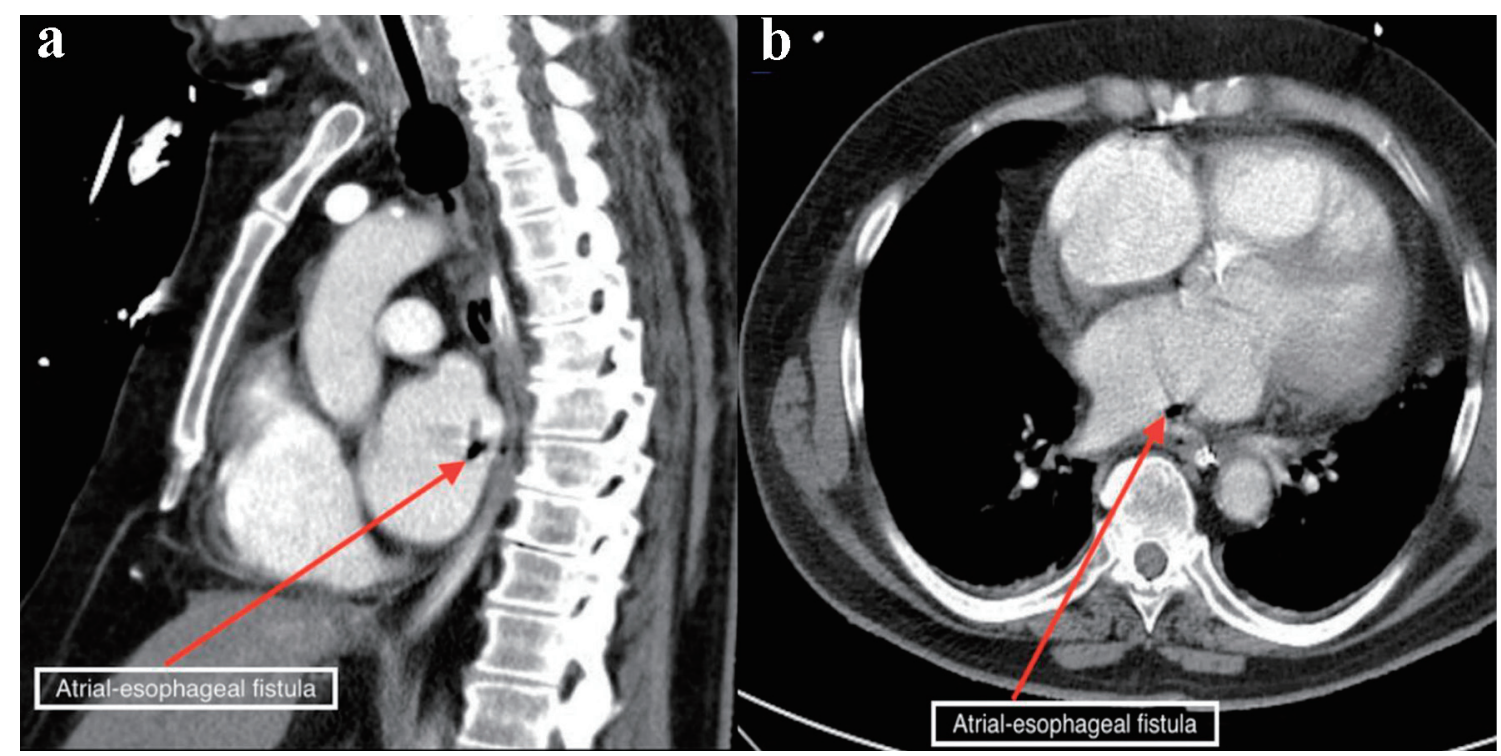

Figure 2. Chest computed tomography scan with contrast showing air contrast (red arrows) within the cavity of the left atrium in the sagittal (a) and axial (b) views.

lator dependency. A multidisciplinary team reached a consensus agreement for palliative measures.

\section{Discussion}

AF is considered to be the most significant cardiac arrhythmia in the USA clinically [4]. Although anti-arrhythmic drugs remain to be the cornerstone for AF management, RFA has become a satisfactory option for resistant cases of AF [4, 5]. RFA for the resistant type of AF is a commonly performed intervention in patients with paroxysmal AF who are categorized as the group of patients who are resistant to anti-arrhythmic drugs [4]. This procedure is usually performed during cardiac surgery as an additional measure or alone as a percutaneous procedure [4]. As per statistics, between 1990 and 2005 in the USA alone 133,000 cases of RFA had been carried out in resistant cases of AF [4]. Every year the number of RFA performed increases at an approximate rate of $15 \%$ [4].

Although there are several documented complications of RFA like pulmonary venous thrombosis, pulmonary venous stenosis, left atrial hematoma, etc., AEF is considered to be one of the most dreadful and fatal complications of RFA [6]. The first documented case of AEF as a complication of RFA was described in the year 2004 by Pappone et al [7]. Since then, there have been many case reports (more than 50) of AEF occurring as a complication of RFA [7-9]. As per the earlier studies, the incidence of AEF was very low following RFA, less than $0.04 \%$. However, recent studies have documented a much higher rate at $0.2-0.4 \%$ [7-9]. It has a mortality rate of $40-80 \%$ [5]. Chest discomfort, cough, reflux symptoms, flu-like symptoms, and severe sepsis are some of the variable clinical presentations associated with this disease [4, 5]. A CT scan of the chest with contrast is regarded as the most reliable non-invasive test for AEF [5].
RFA has a higher success rate between $60 \%$ and $80 \%$ when compared to drug therapy for arrhythmia, but the depth of ablation is rather difficult to control and assess $[10,11]$.

Moreover, the anatomical proximity of the esophagus to the posterior wall of the left atrium (the two vital structures are separated by a thin layer of fat), contributes to the occurrence of AEF following RFA. It is further supported by the fact that around $30-47 \%$ of the patients who undergo RFA suffer from some degree of esophageal mucosal injury, if not a full degree of AEF $[5,11]$.

Several techniques are thought to decrease the risk of thermal injury, like using electroanatomic mapping systems, simultaneous use of intracardiac echocardiography, reduced power delivery, reduced tissue contact pressure with frequent change of probe positions, repeated measurement of the temperature of the esophagus, and fitting the catheter tip with temperature sensors $[11,12]$. Given the fact that all these modifications have not been established conclusively to prevent AEF following RFA, early diagnosis and prompt management play a vital role in saving a patient's life.

Schuring et al published a case series with three cases in elderly males presenting with AEF following RFA. Despite aggressive measures, only one of the patients (case 2) survived, which the authors attributed to the fact that this patient was diagnosed and managed promptly [5]. Dagres et al presented a case series of five cases of AEF following RFA [13]. They noted that only three of the five patients survived and attributed early diagnosis and prompt surgical intervention as the reason behind the favorable outcome [13].

Furthermore, the bidirectional pressure differences between the esophagus and left atrium may lead to an increase in the size of the fistula [14]. If there is more intra-esophageal pressure compared to the intra-atrial pressure, movement of air or bacteria is directed towards the left atrium resulting in air embolism, bacteremia, septic embolism, or endocarditis as 
seen in our case [14]. Vice versa to this, there will be more blood leakage towards the esophagus resulting in gastrointestinal hemorrhage [14]. Therefore, manipulation (nasogastric, orogastric, and transesophageal echocardiography) of the esophagus will result in the widening of this defect and should be contraindicated if AEF is suspected, until after surgery. Our patient presented to the emergency department with bilateral lower extremity weakness, chest discomfort, non-productive cough, and dysphagia 50 days after left pulmonary vein isolation. CT of the chest confirmed AEF, while magnetic resonance imaging of the brain with/without contrast revealed hyperintensity in both cerebral and cerebellar hemispheres, consistent with subacute infarcts suggestive of embolic disease. Despite undergoing aggressive management for his newly diagnosed infective endocarditis and septic emboli, he passed away.

To our knowledge, this case represents one of the few cases documenting life-threatening infective endocarditis with septic emboli as a complication of AEF from RFA. Therefore, clinicians should have a high index of suspicion due to associated grave prognosis.

\section{Learning points}

AEF is a rare complication of RFA. Chest CT scan with contrast has been reported to be the gold standard non-invasive test in diagnosing this iatrogenic defect. Neurologic/cardiac complications of AEF following RFA are rare sequela, and without prompt intervention in most of the cases, the outcome is fatal. Hence increased awareness with rapid diagnosis and immediate surgical intervention are of utmost importance to improve outcomes.

\section{Acknowledgments}

None to declare.

\section{Financial Disclosure}

None to declare.

\section{Conflict of Interest}

None to declare.

\section{Informed Consent}

Informed consent was obtained from the patient before the presentation of this manuscript.

\section{Author Contributions}

Each author has been individually involved in and has made substantial contributions to conceptions and designs, acquisi- tion of data, analysis, interpretation of data, drafting, and editing the manuscript. Ndausung Udongwo contributed to the designs, acquisition of data, analysis, interpretation of data, drafting, and editing of the manuscript. Nusha Fareen contributed to the acquisition of data, drafting, and editing of the manuscript. Temidayo Abe contributed to the designs, interpretation of data, drafting, and editing of the manuscript. Mihir Odak contributed to the drafting and interpretation of data. SmArif Saleh contributed to the drafting and editing of the manuscript. Laith Zamel contributed to the designs, analysis, and editing of the manuscript.

\section{Data Availability}

The authors declare that data supporting the findings of this study are available within the article.

\section{References}

1. Szegedi N, Suhai IF, Perge P, Sallo Z, Hartyanszky I, Merkely B, Geller L. Atrio-esophageal fistula clinically presented as pericardial-esophageal fistula. J Interv Card Electrophysiol. 2021;61(3):623-624.

2. Shim HB, Kim C, Kim HK, Sung K. Successful management of atrio-esophageal fistula after cardiac radiofrequency catheter ablation. Korean J Thorac Cardiovasc Surg. 2013;46(2):142-145.

3. Bailey CW, Tallaksen RJ. Pericardioesophageal fistula following left atrial ablation procedure. J Radiol Case Rep. 2014;8(10):23-31.

4. Kneeland PP, Fang MC. Trends in catheter ablation for atrial fibrillation in the United States. J Hosp Med. 2009;4(7):E1-5.

5. Schuring CA, Mountjoy LJ, Priaulx AB, Schneider RJ, Smith HL, Wall GC, Kadaria D, et al. Atrio-esophageal fistula: a case series and literature review. Am J Case Rep. 2017;18:847-854.

6. Yi F, Hou W, Zhou C, Yin Y, Lu S, Duan C, Cao M, et al. Radiofrequency ablation versus antiarrhythmic drug therapy for atrial fibrillation: meta-analysis of safety and efficacy. J Cardiovasc Pharmacol. 2019;73(4):241-247.

7. Pappone C, Oral H, Santinelli V, Vicedomini G, Lang CC, Manguso F, Torracca L, et al. Atrio-esophageal fistula as a complication of percutaneous transcatheter ablation of atrial fibrillation. Circulation. 2004;109(22):2724-2726.

8. Chavez P, Messerli FH, Casso Dominguez A, Aziz EF, Sichrovsky T, Garcia D, Barrett CD, et al. Atrioesophageal fistula following ablation procedures for atrial fibrillation: systematic review of case reports. Open Heart. 2015;2(1):e000257.

9. Khan MY, Siddiqui WJ, Iyer PS, Dirweesh A, Karabulut N. Left atrial to esophageal fistula: a case report and literature review. Am J Case Rep. 2016;17:814-818.

10. Cappato R, Calkins H, Chen SA, Davies W, Iesaka Y, Kalman J, Kim YH, et al. Updated worldwide survey on the methods, efficacy, and safety of catheter ablation for human atrial fibrillation. Circ Arrhythm Electrophysiol. 
2010;3(1):32-38.

11. Qumseya BJ, Kusumoto F, Wolfsen H. Esophageal injury following left atrial ablation. Gastroenterol Hepatol (N Y). 2012;8(6):414-416.

12. Siegel MO, Parenti DM, Simon GL. Atrial-esophageal fistula after atrial radiofrequency catheter ablation. Clin Infect Dis. 2010;51(1):73-76.

13. Dagres N, Kottkamp H, Piorkowski C, Doll N, Mohr F,
Horlitz M, Kremastinos DT, et al. Rapid detection and successful treatment of esophageal perforation after radiofrequency ablation of atrial fibrillation: lessons from five cases. J Cardiovasc Electrophysiol. 2006;17(11):12131215.

14. He F, Zhang WM, Xu BJ, Huang GP, Chen HD. Atrioesophageal fistula after atrial fibrillation catheter ablation: Acase report. Medicine (Baltimore). 2021;100(2):e24226. 